\title{
Two-Well System under Large Amplitude Periodic Forcing: Stochastic Synchronization, Stochastic Resonance and Stability
}

\author{
Mangal C. Mahato and A.M. Jayannavar \\ Institute of Physics, Sachivalaya Marg, Bhubaneswar-751005, India
}

\begin{abstract}
We study the residence time distributions and explore the possibility of observing stochastic resonance and synchonization of passages in a two-well system driven by a periodic forcing of amplitude larger than a marginal value beyond which one of the two wells become unstable and diasppear. We define and calculate hysteresis loop in the system, the area of which measures the degree of synchronization between the residence time statistics and the input signal, as a function of input noise strength. We analyse the noise induced stability obtained in such a deterministically overall unstable system and within this context discuss the above two phenomena.
\end{abstract}

PACS numbers: $82.20 . \mathrm{Mj}, 05.40 .+\mathrm{j}, 75.60 . \mathrm{Ej}$ 
Stochastic resonance (SR) is a nonlinear phenomena. It helps a system respond to an input signal with an enhanced output signal, as reflected in the power at the same frequency of its spectrum by partially rectifying the noise concomitant to the input signal. This phenomena [1] 6] is observed experimentally [7,9] as well as in numerical [1, 2, 10, 11] and analog simulations [12, 13] as a peak when the output signal to (background) noise ratio (SNR) in the power spectrum is plotted as a function of input noise strength. This could find practical application in detecting weak signals and understandably theoretical treatments and numerical simulations, except in a few cases [14,15, are mostly focussed on small amplitude periodic input signals. In this work we study a two-well system with large (overcritical) amplitude periodic forcing (input signal) aided by zero-mean Gaussian white noise and explore the possibility and physical origin of observing SR in the distribution of residence times of a Brownian particle in any one of the two wells. SR is observed in a well defined range of sweep rates of the input signal. We find that $\mathrm{SR}$ in this case is made possible by noise-affected slowing down of the passages of the particle from an unstable well to the stable well of the two-well system. Also, this slowing down has marked effect on the synchronization of passages. This will be discussed below after describing the procedure of our calculation.

We represent the two-well system by the usual Landau potential

$$
U(m)=-\frac{a}{2} m^{2}+\frac{b}{4} m^{4}
$$

and solve the overdamped Langevin equation

$$
\dot{m}=-\frac{\partial U(m)}{\partial m}+h(t)+\hat{f}(t)
$$

for the motion $m(t)$, when the system is subjected to a time dependent external forcing $h(t)$ and a Gaussian random fluctuating force $\hat{f}(t)$ with statistics $<\hat{f}(t)>=0$ and $<$ $\hat{f}(t) \hat{f}\left(t^{\prime}\right)>=2 D \delta\left(t-t^{\prime}\right)$. We take $a=2.0$ and $b=1.0$ throughout our calculation and $h(t)$ to be a saw-tooth type external periodic field with amplitude $h_{0}=1.4 h_{c}$, where $\left|h_{c}\right|$ is the minimum value of field at which one of the two wells of $\Phi(m)=U(m)-m h(t)$ become 
unstable and then diassapear. We set $m(t=0)$ in one of the two well minima (the initial condition is not really important) and monitor the time evolution $m(t)$ for a long time (corresponding to a large number of passages) to obtain meaningful average values. If the particle at some instance $t$ is in a well, we say the passage to take place at a later time to the other well only when the trajectory $m(t)$ traverses across the inflection point on to the other side of the potential barrier separating the first well from the other. We put markers on the time axis whenever a passage takes place, the interval between two consecutive markers giving the residence time in a well. We thus obtain the residence time distributions $\rho_{1}(\tau)$ and $\rho_{2}(\tau)$ in the two wells respectively. (In our present case the two distributions should be identical.) The markers on the time axis also give the field values $h(t)$ at which passage takes place and thus the jump field distributions $\rho_{12}(h)$ and $\rho_{21}(h)$ are obtained for passages from well 1 to 2 , and from well 2 to 1 , respectively. These distributions, $\rho_{i}(t)$ and $\rho_{i j}(h)$ are used below in our discussion of SR (by calculating the SNR from the power spectrum [17] of $\rho_{i}$ 's) and stochastic synchronization (SS)(by calculating the hysteresis loop area) in the two-well system at large forcing amplitude $h_{0}=1.4 h_{c}$. As $h(t)$ varies periodically, the left well, for example, becomes unstable and disappears for the part of the period for which $h(t) \geq h_{c}$ and so is the other well for the part of the period for which $h(t) \leq-h_{c}$. During these two intervals (due to the nonexistence of the intervening potential barrier) passage to the stable well is possible even when $D=0$. For small sweep rates $|\dot{h}|$, of $h(t)$, one passage from one well to the other per period is guaranteed for $D=0$. However, for large $|\dot{h}|$ the passage may be delayed and the distribution $\rho_{i}(t)$ may spill over to the next and even later periods of $h(t)$. The multipeaked $\rho_{i}(t)$ indicates the possibility of SR at large sweep rates as $D$ is gradually increased. And, indeed, we find the system to show stochastic resonant behaviour.

The exact value of $D$ at which the stochastic resonance occurs is difficult to pinpoint as the uncertainties involved in measuring the output noise level is large. However, the occurrence of resonant behaviour can be seen clearly from Fig. 1, where we plot the power spectral density (of $\rho(t)$ ) for various $D$ values: Almost overlapping broad peakes with ap- 
preciable height appear only for a small intermediate range of $D$ values. One may, however, ask whether this is not an artefact of having large sweep rates and, thus, having no relation with real physical situations. This doubt can be set to rest once we look into an another important question of noise induced stability of "unstable states".

As stated earlier, for small enough $|\dot{h}|$, the average number of passages per cycles (ANPPC) of $h(t)$ at $D=0$ is 1 but starts decreasing sharply to zero as $|\dot{h}|$ is increased beyond a threshold value $\dot{h}_{t h}$ (in the present case $\approx 0.384 h_{c}$ ) as shown in Fig. 2 . Hence till $|\dot{h}|=\dot{h}_{t h}$ the sweep rate is such that the particle gets enough time to roll down to the stable well in each and every cycle in the absence of friction (noise).Now, let us look at the variation of ANPPC of $h(t)$ as a function of $D$ for various $|\dot{h}|$ [Fig. 3]. The curves for $|\dot{h}|<\dot{h}_{t h}$ show that as the noise is switched on ANPPC decreases to less than 1, attains a minimum and then rises to 1 and beyond 1 corresponding to processes dominated by noise. This is an important observation and shows clearly that the presence of noise actually slows down the decay of the deterministically overall unstable states in an appropriate range of $|\dot{h}|$ and $D$ values. This fact has been reported earlier [14, 16] too in other systems. In order to make our discussion on SR sensible we need, therefore, to restrict $|\dot{h}|$ to the maximum value of $\dot{h}_{t h}$. Fig. 1 corresponds to $\dot{h}=0.28 h_{c}$ which is well within the range, and the observation of SR in the case of large amplitude periodic forcings can, thus, safely be considered to be genuine and not an artefact of large sweep rates. Having discussed about the SR behaviour reflected in the residence time distribution we ask the question of synchronization property of the passages with reference to the input signal (periodic forcing).

The phase relationship of passages with the input signal is well reflected in the distributions $\rho_{i j}(h)$ of field values $h(t)$ at which passages take place from well $i$ to well $j$. In order to obtain a quantitative relationship we invoke the hysteretic property of the system. The hysteresis loop is defined here as the difference, $\bar{m}(h)$, of relative populations $m_{1}(h)$ and $m_{2}(h)$ in the two wells 1 and 2 , respectively. Here $m_{2}(h)$ is calculated from the discrete equation, 


$$
m_{2}(h)=m_{2}(h-\Delta h)-m_{2}(h-\Delta h) \rho_{21}(h) \Delta h+m_{1}(h-\Delta h) \rho_{12}(h) \Delta h,
$$

with negligibly small $\Delta h$, and similarly for $m_{1}(h)=1-m_{2}(h)$ and with the condition of closure of the loop in a cycle of $h(t)$. The area of the hysteresis loop is a good measure of the degree of synchronization of passages. For instance, if the passages from well 1 to the well 2 take place only at $h=h_{0}$, where the barrier to passage $1 \rightarrow 2$ is minimum, and similarly from well 2 to well 1 only at $h=-h_{0}$ (a case of maximum synchronization) then the hysteresis loop is a rectangle with the largest area, whereas if the passages take place all over randomly and uniformly from $h_{0}$ to $-h_{0}$, we have the least area, equal to zero, a case of least synchronization between passages and the input periodic signal. On plotting the hysteresis loop area as a function of $D$ for various $|\dot{h}|$ (Fig. 4), we find that the area peaks in the range of $D$ where we find noise induced delay of passages. However, these peak positions, in general, do not coincide with the peak positions of SNR. This stochastic synchronization of passages may not, however, be entirely unrelated to stochastic resonance [18].

In conclusion, we observe that SR is a meaningful phenomena at large amplitude periodic forcings too. It is because of the presence of noise that the particle stochastically fails, in some of the field sweep cycles, to roll down to the stable well so that the residence time distribution contains more than one peak. For a lone peak in the residence time distribution may not yield SR. Noise of appropriate strength prolongs the decay of the deterministically overall unstable state and thereby it helps to maximize the degree of synchronization of passages (with respect to the externally applied periodic field) in the two-well system. 


\section{REFERENCES}

[1] A.R. Benzi, A. Sutera, and A. Vulpiani, J. Phys.: Math. Gen. 14, L453(1981).

[2] B. McNamara and K. Wiesenfeld, Phys. Rev. Abf 39, 4854(1984).

[3] P. Jung ang P. Hänggi, Europhys. Lett. 8, 505 (1989); Phys. Rev. A44, 8032(1991).

[4] L. Gammaitoni, F. Marchesoni, and S. Santucci, Phys. Rev. Lett. 74, 1052(1995), and references therein.

[5] R.F. Fox, Phys. Rev. A39, 4148(1989).

[6] Proceedings of the NATO ARW Stochastic Resonance in Physics and Biology, edited by F. Moss, A. Bulsara, and M.F. Shlesinger [J. Stat. Phys. 70, No. 1(1993)].

[7] S. Fauve and F. Heslot, Phys. Lett. 97A, 5(1983).

[8] B. McNamara, K. Wiesenfeld, and R. Roy, Phys Rev. Lett. 60, 2626(1988); G. Vemuri and R. Roy, Phys. Rev. A39, 4668(1989).

[9] J.K. Douglass, L. Wilkens, E. Pantazelou, and F. Moss, Nature 365, 337(1993).

[10] J.M. Casado and M. Morillo, Phys. Rev. E49, 1136(1994).

[11] M.C. Mahato and S.R. Shenoy, Phys. Rev. E50, 2503(1994).

[12] L. Gammaitoni, F. Marchesoni, E. Menichell-Saetta, and S. Santucci, Phys. Rev. Lett. 62, 349(1989).

[13] G. Debnath, T. Zhou, and F. Moss, Phys. Rev. A39, 4323(1989); T. Zhou and F. Moss, ibid 41, 4255(1990); T. Zhou, F. Moss, and P. Jung, ibid 42, 3161(1990).

[14] I. Dayan, M. Gitterman, and G.H. Weiss, Phys. Rev. A46, 757(1991)

[15] P. Jung and P. Hänggi, Phys. Rev. A44, 8032(1991)

[16] R.N. Mantegna and B. Spagnolo, Phys. Rev. Lett. 76, 563(1996). 
[17] W.H. Press, S.A. Teukolsky, W.T. Vetterling, and B.P. Flannery, Numerical Recipes (in Fortran):The Art of Scientific Computing, Cambridge Univ. Press, 1992, pp. 542.

[18] M.C. Mahato and A.M. Jayannavar, unpublished. 
FIG. 1. Shows power spectral density (psd) for three values of $D$ : (a) $D=.001$ (dotted), (b) $D=.1$ (solid line), and (c) $D=.4$ (dash-dotted), at $\dot{h}=.028 h_{c}$. We have taken, for each curve, 256 (augmented with zeroes) data points at a time interval of 2.0.

FIG. 2. Average number of passages per cycle (ANPPC) is plotted as a function of $\dot{h}$ for $D=0.00001$. Averaging is done over 5000 cycles. The line is drawn to guide the eye.

FIG. 3. Shows ANPPC as a function of $D$ for various $\dot{h}$ values:(a) $0.05 h_{c}(\circ),(\mathrm{b}) 0.2 h_{c}(\square)$, (c) $0.28 h_{c}(\diamond),(\mathrm{d}) 0.35 h_{c}(\triangle)$, and (e) $0.4 h_{c}(\triangleleft)$. For $\dot{h} \widetilde{>} 0.392 h_{c}$ the curves, for example the curve for $\dot{h}=.4 h_{c}$, show monotonic behaviour starting from $\mathrm{ANPPC}=0$. The lines are drawn to guide the eye.

FIG. 4. Hysteresis loop area $A$ versus $D$ for various $\dot{h}$ values:(a) $0.05 h_{c}\left(\right.$ o), (b) $0.2 h_{c}(\square),(\mathrm{c})$ $0.28 h_{c}(\diamond),(\mathrm{d}) 0.35 h_{c}(\triangle)$, and $(\mathrm{e}) 0.4 h_{c}(\triangleleft)$. The lines are drawn to guide the eye. 